\section{Spasmophilia and entrapment nerve syndrome comorbidities in fibromyalgic patients: a possible neuromuscular pain generator}

\author{
F. Ginanneschi', G. Filippou², A. Adinolfi², V. Di Sabatino², \\ B. Frediani ${ }^{2}$, A. Rossi ${ }^{1}$ \\ ${ }^{2}$ Department of Medical, Surgical and Neurological Sciences, Neurology \\ and Clinical Neurophysiology Section, University of Siena, Italy; \\ ${ }^{1}$ Department of Medical, Surgical and Neurological Sciences, Rheumatology Section, University of Siena
}

\section{SUMMARY}

This paper is aimed at investigating whether peripheral dysfunction at the neuromuscular level may represent a pain generator in fibromyalgia.

We studied the prevalence of spasmophilia (SP), carpal tunnel syndrome (CTS) and ulnar neuropathy at the elbow (UNE) in a group of 40 subjects suffering from fibromyalgia. Clinical and electrophysiological data were obtained to ascertain whether comorbid conditions were present. For subjective evaluation of symptoms severity, validated questionnaires for CTS and UNE were completed by patients. Twenty subjects were positive for SP (50\%); CTS was diagnosed in 12 subjects (30\%); no patient suffered from UNE; 6 subjects were affected at the same time by SP and CTS (15\%); 14 subjects (35\%) were affected by SP alone. The prevalence of CTS and $\mathrm{SP}$ was higher in fibromyalgia subjects than in the general population. The scores of the questionnaires related to CTS were significantly higher in fibromyalgia subjects positive for CTS, with respect to the other subjects. In fibromyalgia, CTS and SP may be considered clinical entities in themselves, the importance of which lies in their acting as peripheral pain generators that enhance or initiate central sensitization, thereby contributing to chronic widespread pain. The amplification of pain is indeed a correctable/misguided message that occurs inside the brain of fibromyalgia subjects and identification and local treatment of pain generators would lessen the total pain burden. The magnitude of the overlap in symptoms between fibromyalgia and CTS/SP necessitates careful investigation of these conditions.

Key words: Carpal tunnel syndrome; Fibromyalgia; Latent tetany; Ulnar neuropathy.

\section{INTRODUCTION}

ubjects developing fibromyalgia commonly have a lifelong history of chronic pain throughout their body with concomitant depression and sleep disorder; they are likely to have a history of headaches, dysmenorrhea, temporomandibular joint disorder, functional gastrointestinal and urinary disorders, endometriosis, and other regional pain syndromes (1). The etiology of fibromyalgia is still unclear. Several factors such as dysfunction of the central and autonomic nervous systems, neurotransmitters, hormones, immune system, external stressors, psychiatric aspects, and others seem to be involved.

Although central sensitization is considered to be the main mechanism involved, a contribution by peripheral pain generators to the syndrome is, however, being increasingly recognized $(1,2)$. In effect, fibromyalgia patients present a significantly higher frequency of numerous painful focal disorders with respect to healthy subjects (3). The relationship of the comorbid conditions to fibromyalgia has not been clarified. They can be considered as the
Corresponding author: Federica Ginanneschi

Department of Medical, Surgical and Neurological Sciences; Neurology-Neurophysiology Unit, University of Siena. Policlinico Le Scotte Viale Bracci, 1 - 53100 Siena, Italy E-mail: ginanneschi@unisi.it 
result of hyper-effective ascending central pain transmission resulting from deficient descending pain inhibition, or they can be considered as clinical entities in themselves, whose importance in fibromyalgia lies in their acting as peripheral pain generators that enhance or initiate central sensitization.

The present paper is aimed at investigating whether peripheral dysfunction at the neuromuscular level may represent a pain generator in fibromyalgia. We studied the prevalence of neuromuscular hyperexcitability syndrome (spasmophilia), carpal tunnel syndrome (CTS) and ulnar neuropathy at the elbow (UNE) comorbidity in 40 fibromyalgia patients. Some of the wide range of symptoms of fibromyalgia, such as pain and paresthesia, are indeed common in compressive peripheral neuropathies and spasmophilia (SP), so one disease may mask the other.

\section{MATERIALS AND METHODS}

\section{Subjects}

A prospective study was carried out on 40

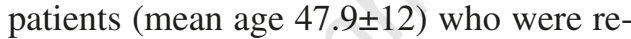
ferred to the Rheumatology Unit of Siena from January to December 2012 with a diagnosis of fibromyalgia according to the 1990 and 2010 American College Rheumatology criteria. All patients were female. We excluded all subjects who had undergone surgery of the upper limb, as well as those with radiculopathy and plexopathy of the arm, polyneuropathy, previous arm trauma, diabetes, rheumatic or thyroid diseases, renal failure, history of alcoholism and central nervous system diseases, malignancy in the previous 5 years, and/or previous intake of medication considered toxic to the peripheral nervous system. All 40 fibromyalgia patients underwent clinical examination and electrophysiological studies to ascertain whether comorbid conditions, specifically CTS, UNE and SP, were present in these subjects. One investigator carried out neurophysiological studies.

Finally, we enrolled a control group of 40 healthy volunteers matched for sex and age with the fibromyalgia population, to ex- plore the prevalence of the SP in a healthy population. The latter data is indeed almost entirely lacking in available literature. There is only one paper from 1986, which reported an SP prevalence of $17 \%$ in the healthy population (4).

\section{Diagnostic criteria for carpal tunnel syndrome}

Carpal tunnel syndrome was diagnosed according to the American Academy of Neurology criteria (5), which include clinical history, symptoms and neurographic evidence of slowing of distal median nerve conduction velocity. Clinical history and symptoms included any kind of paresthesia and/or pain in all or part of the hand innervated by the median nerve. The electrophysiological examinations were conducted on 80 hands belonging to all 40 fibromyalgia subjects. Surface recording electrodes were placed over the motor point of the abductor pollicis brevis (APB) muscle; distal motor latency (DML) was measured with a distance of $7 \mathrm{~cm}$ between the stimulation point of the nerves at the wrist and the APB muscles. A rubber-suction surface recording electrode placed on the wrist and stimulating ring electrodes placed on the fingers were used for median sensory conduction studies. Maximum orthodromic sensory conduction velocity (SCV) was calculated from the onset of the negative peak, and the responses were averaged. Maximum sensory nerve action potential amplitude (SNAPa) of the median (M3, middle finger-wrist; M4, ring fingerwrist) nerve was determined.

The minimum electrophysiological criteria to confirm the clinical diagnosis of CTS were prolonged DML ( $\geq 4.4 \mathrm{~ms}$ ) and/or abnormal SCV of the median nerve $(\leq 45.2 \mathrm{~m} / \mathrm{s}$ for M3 and $\leq 43 \mathrm{~m} / \mathrm{s}$ for M4). When absolute values of $\mathrm{SCV}$ were normal in the digit-wrist segment, a difference of $10 \mathrm{~m} / \mathrm{s}$ between SCVs of ulnar nerve (U4, ring finger-wrist) and M4 was considered significant.

\section{Diagnostic criteria for ulnar neuropathy at the elbow}

The electrophysiological examinations were conducted on 80 hands belonging to all 40 
fibromyalgia subjects. Surface recording electrodes were placed over the motor point of the abductor digiti minimi (ADM) muscle; motor conduction velocity (MCV) was calculated from below the elbow to the wrist and below the elbow to above the elbow (10 $\mathrm{cm}$ across-elbow distances); DML was measured with a distance of $7 \mathrm{~cm}$ between the stimulation point of the nerve at the wrist and the ADM; compound muscle action potential amplitude (CMAPa) was also measured from baseline to the negative peak. A rubber-suction surface recording electrode placed on the wrist and stimulating ring electrodes placed on the fingers were used for sensory conduction studies. Maximum SCV was calculated from the onset of the negative peak, and the responses were averaged. Maximum SNAPa of the U4 and U5 (little finger-wrist) was determined. Needle electromyography of ADM and first dorsal interosseous muscles was performed.

Diagnosis of UNE was based on the coexistence of:

1) clinical history and symptoms suggesting UNE: numbness and paraesthesia or pain in the part of the hand innervated by the ulnar nerve; pain at the elbow; motor symptoms might be absent or ranged from mild clumsiness to severe weakness of the hand;

2) electrophysiological criteria: reduction of MCV from above elbow to below elbow (MCV less than $50 \mathrm{~m} / \mathrm{s}$ ); MCV from above to below elbow more than $10 \mathrm{~m} / \mathrm{s}$ slower than MCV from below elbow to wrist; decrease in CMAPa greater than $20 \%$ between stimulation above and below elbow; reduction of ulnar nerve SNAPa from U5; abnormal electromyography of ulnar hand muscles. These methods were in accordance with the guidelines of the American Academy of Electrodiagnostic Medicine (6).

\section{Diagnostic criteria for spasmophilia}

Spasmophilia diagnosis was based on electromyographic criteria. A concentric needle electrode was placed on the first dorsal interosseous muscle of one hand, then using a sphygmomanometer cuff, a pres- sure greater than $20 \mathrm{mmHg}$ of the patient's systolic blood pressure was applied to the arm and maintained for 8 minutes. Electromyographic spontaneous activity was recorded during this time, for 5 minutes after decompression of the cuff and then during hyperpnea for 3 minutes. Diagnosis of SP was considered in the presence of spontaneous doublets, triplets and multiplets either during/after ischemia or during/after hyperpnea. The same investigator carried out neurophysiological studies both on fibromyalgia subjects and on the control population.

\section{Self-evaluation of symptoms and functional status of hand questionnaires}

For subjective evaluation of severity of symptoms, the Italian version of the Boston Questionnaire (BQ) (7) and UNE Questionnaire (UNEQ) (8) were completed by the 40 fibromyalgia patients. The BQ and UNEQ were validated to measure subjective discomfort experienced by CTS and UNE patients, respectively. The BQ is divided into two parts. The first part (11 items) evaluates symptoms (BQS), and the second part (8 items) evaluates the functional status of the hand (BQM). The UNEQ is composed of nine questions and it considers symptoms (numbness and tingling) of the fourth and fifth fingers, possible elbow pain and changes in these symptoms in relation to elbow position. Hand weakness is also evaluated. Each question of UNEQ and BQ has five possible answers scored from 1 to 5 , according to severity of symptoms or the difficulty of performing a certain activity. Each score is calculated as the mean of the score of the individual items. Severe impairment is indicated by a high score.

\section{Statistical analysis}

Data was tested for normality (Kolmogorov-Smirnov distance method). Values in the groups were compared using one-way analysis of variance (ANOVA). If comparison of groups showed significant differences, Bonferroni's multiple comparison test was performed. Statistical significance was assumed if the $p$ value was $<0.05$. Sta- 
tistical difference in the frequency of SP between fibromyalgia patients and controls were calculated with Fisher's exact test. Results are presented as mean values and standard deviation (SD).

\section{RESULTS}

All 40 fibromyalgia patients were examined. Carpal tunnel syndrome was diagnosed in 12 subjects $(30 \%)$. No patient was suffering from UNE. Twenty subjects resulted positive for SP (50\%), while the prevalence of SP in the control group was $10 \%$ (4 out 40 subjects). Six patients were affected at the same time by SP and CTS $(15 \%) ; 14(35 \%)$ were affected by SP alone. Carpal tunnel syndrome severity was similar between the latter and the former group.

Table I shows demographic data and results of questionnaires administered to patients. There are no significant differences between groups for UNEQ. Conversely, BQ scores were higher in subjects with CTS comorbidity. In particular, both BQS and BQM scores were significantly higher in fibromyalgia subjects affected by CTS, with respect to all the other subjects (that is, the fibromyalgia subjects without comorbidity and/or positive for SP alone).

The prevalence of SP in the healthy population was $10 \%$ (4 out 40 subjects). There was a significant difference in the frequency of SP between fibromyalgia patients and controls $(\mathrm{p}<0.0002)$.

\section{DISCUSSION}

The mechanism of sensory symptoms in fibromyalgia has not been exactly elucidated and it is mainly considered to be a result of abnormal sensory perception. In effect, a recent review stated that fibromyalgia could be thought of as a centralized pain state; centralized refers to pain originating in or amplified by the central nervous system (1). This belief does not imply that peripheral nociceptive/neuropathic input is not contributing to pain in fibromyalgia subjects.

Some of the wide range of symptoms of fibromyalgia, such as pain and paraesthesia are common in compressive peripheral neuropathies and SP so that one disease may mask the other. In the present paper we have studied the prevalence of three common pathological conditions that cause paraesthesia and/or pain, namely CTS, UNE and SP, in 40 fibromyalgia patients. The results demonstrate a high prevalence of SP (50\%) and CTS (30\%); conversely, no patient was affected by UNE.

The prevalence of CTS is significantly higher in fibromyalgia subjects than in the general population (2.7\%) (9). The

Table I - Demographic data, questionnaires results and differences between the groups.

\begin{tabular}{|c|c|c|c|c|c|c|c|}
\hline $\begin{array}{c}\text { Total FM } \\
\text { no. 40 }\end{array}$ & $\begin{array}{c}\text { Negative } \\
\text { (no. 15) }\end{array}$ & $\begin{array}{c}\text { CTS tot } \\
\text { (no. 12) }\end{array}$ & $\begin{array}{c}\text { SP tot } \\
\text { (no. 20) }\end{array}$ & $\begin{array}{c}\text { CTS+SP } \\
\text { (no. 6) }\end{array}$ & $\begin{array}{c}\text { SP alone } \\
\text { (no. 14) }\end{array}$ & p values & $\begin{array}{c}\text { Bonferroni's Multiple } \\
\text { Comparison Test }\end{array}$ \\
\hline Age (years) & $46.6 \pm 11.3$ & $51.8 \pm 11.7$ & $50.8 \pm 11$ & $50.82 \pm 10$ & $46.1 \pm 15$ & n.s. & $\begin{array}{c}\text { Negative vs CTS tot } \\
\text { Negative vs CTS+SP* } \\
\text { SP only vs CTS tot }^{*}\end{array}$ \\
\hline BQS & $2.54 \pm 0.68$ & $3.5 \pm 0.65$ & $2.99 \pm 0.83$ & $3.67 \pm 0.57$ & $2.59 \pm 0.7$ & $p=0.001$ & $\begin{array}{c}\text { Negative vs CTS tot } \\
\text { Negative vs CTS+SP* } \\
\text { SP only vs CTS tot }\end{array}$ \\
\hline BQM & $2.21 \pm 0.64$ & $3.4 \pm 0.8$ & $2.86 \pm 1.07$ & $3.68 \pm 0.7$ & $2.46 \pm 0.95$ & $p=0.003$ & n.s. \\
\hline UNEQ & $2.1 \pm 0.75$ & $2.85 \pm 0.9$ & $2.45 \pm 0.9$ & $3.1 \pm 0.85$ & $2.06 \pm 0.7 .5$ & & \\
\hline
\end{tabular}

BQF: Boston questionnaire functional; BQS: Boston questionnaire symptoms; CTS: carpal tunnel syndrome; CTS total: FM subjects with CTS comorbidity; CTS+SP: FM subjects positive for both CTS and SP; FM: fibromyalgia; Negative: FM subjects without comorbidities; no: number; n.s.: not significant; SP: spasmophilia; SP alone: FM subjects with SP comorbidity alone; SP total: FM subjects positive for SP alone and/or SP and CTS; Total FM: all fibromyalgia subjects, irrespective of the comorbidities; UNEQ: questionnaire ulnar neuropathy elbow.

Results are reported as mean values \pm standard deviation. Multiple comparison test: ${ }^{*} 0.05$ (the results of the post hoc Bonferroni test are reported only when significant). 
BQ scores were abnormal only in CTS subjects whereas there are no significant differences between groups for UNEQ. The self-administered questionnaires BQ and UNEQ are a way to assess patient discomfort and represent a reliable and reproducible instrument to evaluate CTS and UNE symptoms, respectively $(7,8)$. The strong association between CTS and a high BQ score in fibromyalgia patients emphasizes the fact that CTS is a crucial cause of impairment in these patients. This means that sensory symptoms reported by fibromyalgia subjects were, at least in part, related to a specific peripheral pain generator. In reality, many papers report that the prevalence of CTS in fibromyalgia is higher than in the general population (10-14). Conversely, data on UNE prevalence in fibromyalgia are very rare. Only one paper analyzed peripheral nerve conduction in 33 fibromyalgia subjects and none had ulnar nerve conduction failure (13). The high prevalence of CTS in the fibromyalgia population suggests that common underlying mechanisms are at work. At present, there is no study explaining the mechanisms why CTS is frequently observed in patients with fibromyalgia.

Regarding SP, in contrast to CTS, epidemiologic data concerning the general population are very rare.

In the available literature only one paper has explored the prevalence of SP in healthy individuals, reporting a $17 \%$ prevalence (4), while in the present paper we report a prevalence of $10 \%$. Both percentages are lower than that reported in fibromyalgia populations by Bazzichi et al. (15) (25.8\% of cases) and by us (50\% of cases), suggesting a comorbidity between fibromyalgia and SP. The basis of neuromuscular hyperexcitability in SP is thought to be a disturbance of calcium and magnesium metabolism. From the study by Bazzichi et al., (15) significant differences emerge between the patients affected by fibromyalgia alone and the fibromyalgia group positive for SP: the latter group had lower levels of intracellular magnesium compared to fibromyal- gia patients negative for SP, suggesting a relationship between SP and dysfunction at the neuromuscular level.

\section{CONCLUSIONS}

In conclusion, some neuromuscular diseases, such CTS and SP, are associated conditions in fibromyalgia subjects, representing peripheral pain generators in fibromyalgia. In reality, although fibromyalgia is generally not thought to be caused by peripheral damage or inflammation, some evidence exists supporting treatment of peripheral pain generators as considered factors driving central sensitization (1). The amplification of pain is indeed a correctable/misguided message that occurs inside the brain of fibromyalgia subjects and the identification and the local treatment of pain generators lessens the total pain burden and facilitates rehabilitation $(3,16)$. This is particularly important for a syndrome like fibromyalgia where management options available so far are still of limited effectiveness in a large percentage of cases (17).

The key message of our report is that the magnitude of the association between fibromyalgia and CTS/SP requires a thoughtful search for these pain generators since their presence may be masked by the symptoms of fibromyalgia.

\section{Conflicts of interest: none}

\section{REFERENCES}

1. Clauw DJ. Fibromyalgia. A Clinical Review. JAMA 2014; 311: 1547-55.

2. Arnold LM. Strategies for managing fibromyalgia. Am J Med. 2009; 122: S31-43.

3. Affaitati G, Costantini R, Fabrizio A, et al. Effects of treatment of peripheral pain generators in fibromyalgia patients. Eur J Pain. 2011; 15: 61-69.

4. de Romanis F, Feliciani M, Rosati MV, et al. Spasmophilia: a clinical neurophysiopathological and biochemical study. Funct Neurol. 1987; 2: 239-246.

5. American Academy of Electrodiagnostic Medicine Quality Assurance Committee. Literature review of the usefulness of nerve conduction studies and electromyography for the 
evaluation of patients with carpal tunnel syndrome. Muscle Nerve 1993; 16: 1392-414.

6. American Association of Electrodiagnostic Medicine Quality Assurance Committee: Practice parameter. Electrodiagnostic studies in ulnar neuropathy at the elbow. Neurology 1999; 52:688-90.

7. Padua R, Padua L, Romanini E, et al. Boston Carpal Tunnel Questionnaire Italian version. Ital J Orthop Traumatol. 1998; 24: 123-9.

8. Mondelli M, Padua L, Giannini F, et al. A selfadministered questionnaire of ulnar neuropathy at the elbow. Neurol Sci. 2006; 27: 402-11.

9. Atroshi I, Gummesson C, Johnsson R, et al. Prevalence of carpal tunnel syndrome in a general population. JAMA. 1999; 282: 153-8.

10. Perez-Ruiz F, Calabozo M, Alonso-Ruiz A, et al. High prevalence of undetected carpal tunnel syndrome in patients with fibromyalgia syndrome. J Rheumatol. 1995; 22: 501-4.

11. Cimmino MA, Parisi M, Moggiana G, Accardo $\mathrm{S}$. The association between fibromyalgia and carpal tunnel syndrome in the general population. Ann Rheum Dis. 1996; 55: 780.

12. Sarmer S, Yavuzer G, Küçükdeveci A, Ergin S. Prevalence of carpal tunnel syndrome in patients with fibromyalgia. Rheumatol Int. 2002; 22: 68-70.

13. Ersoz M. Nerve conduction tests in patients with fibromyalgia: comparison with normal controls. Rheumatol Int. 2003; 23: 166-70.

14. Nacir B, Genc H, Duyur Cakit B, et al. Evaluation of upper extremity nerve conduction velocities and the relationship between fibromyalgia and carpal tunnel syndrome. Arch Med Res. 2012; 43: 369-74.

15. Bazzichi L, Consensi A, Rossi A, et al. Spasmophilia comorbidity in fibromyalgia syndrome. Clin Exp Reumatol. 2010; 28: S94-9.

16. Borg-Stein J. Management of peripheral pain generators in fibromyalgia. Rheum Dis Clin North Am. 2002; 28: 305-317.

17. Carville SF, Arendt-Nielsen S, Bliddal H, et al. EULAR evidence based recommendations for the management of fibromyalgia syndrome. Ann Rheum Dis. 2008; 67: 536-41. 